\title{
El Comité Mudaliar (1962): evaluación de los comienzos
}

\section{The Mudaliar Committee (1962): Assessing the beginnings}

\author{
LAURA CARBALLIDO CORIA \\ Universidad Autónoma Metropolitana-Cuajimalpa, México
}

Resumen: El Comité Mudaliar fue integrado en los primeros años del periodo poscolonial en India para evaluar la salud de la población y hacer recomendaciones. Buscó producir conocimiento que legitimara al Estado y sirviera de guía en la definición de la salud pública. Mudaliar manifestó tener una postura crítica, pero el reporte que produjo junto con el resto del comité estuvo marcado por limitantes y contradicciones. Al estudiar este proceso, busco contribuir a una mejor comprensión de los primeros años del periodo poscolonial y mostrar que el paso del dominio colonial a la independencia no se reduce a rupturas absolutas ni a continuidades sencillas.

Palabras clave: comités y comisiones de investigación; India independiente; salud pública; expertos.

Abstract: The Mudaliar Committee was set up in the early years of postcolonial India to assess the population's health and to make

Recepción: 13 de diciembre de 2019. / Aceptación: 19 de marzo de 2020. 
recommendations. It sought to generate knowledge to grant legitimacy to the State and inform public health policy-making. Mudaliar professed that he was highly critical, yet the report produced by him and the Committee's other members had several limitations and contradictions. In analyzing this process, the author seeks to improve our understanding of the early postcolonial period, and to show that the shift from colonial rule to independence cannot be simplified as a clean break or as seamless, straightforward continuities.

Keywords: committees and commissions of enquiry; independent India; public health; experts.

En 1959, el Ministerio de Salud de India instituyó el Comité de Investigación sobre Salud y Planeación (Health Survey and Planning Committee), también conocido como Comité Mudaliar, para analizar la salud pública de los primeros años de India independiente, tomando como punto de partida el último estudio realizado por la administración colonial (Comité Bhore, 1943-1946). Debía evaluar los resultados del primer y del segundo planes quinquenales en cuanto a salud y hacer recomendaciones para un tercero. El comité estaría dirigido por el doctor A. Lakshmanaswami Mudaliar, quien había ocupado cargos importantes: rector de la Universidad de Madrás y director del Colegio Médico de Madrás. Además, se trataba de una figura internacional prominente, pues había representado a India en la Primera Asamblea de la Salud Mundial en Ginebra, en 1948, y dirigió el Comité Ejecutivo de la Organización Mundial de la Salud (OMs). En esta ocasión, Mudaliar encabezaría un comité integrado por un grupo de expertos en salud pública y medicina. ${ }^{1}$

Los comités y las comisiones de investigación ad hoc figuraron de manera destacada en el periodo colonial y fueron muy utilizados a partir de la década de 1830 para tratar diversos temas, especialmente los relacionados con política pública. En el periodo poscolonial, este mecanismo ha sido usado

${ }^{1}$ Agradezco las sugerencias de los dictaminadores. 
con propósitos similares: para realizar investigaciones sobre temas específicos y hacer recomendaciones al respecto. ${ }^{2} \mathrm{La}$ Comisión de Reorganización de los Estados (1953), la Comisión Mandal (1979) y el Comité Sachar (2005) son ejemplos de esto.

Este trabajo plantea examinar de manera crítica dos elementos del Comité Mudaliar: primero, el tipo de integrantes que tuvo y que refleja el trabajo de los "expertos", y segundo, algunas recomendaciones contenidas en el reporte, cuyas más de 500 páginas aspiraban a condensar el conocimiento correspondiente a la salud y las condiciones de vida en India en $1962 .^{3}$ En conjunto, estos dos elementos permiten un acercamiento a la forma en que se percibía la salud.

Con esto espero contribuir, por un lado, a entender mejor un mecanismo que ha sido usado por varios Estados en el pasado y en el presente, no sólo en la Mancomunidad británica, sino también en otras regiones del mundo. ${ }^{4}$ Las comisiones y los comités son generalmente vistos como fuentes ideales para los historiadores, pero es importante analizarlos con cuidado. Son parte de los instrumentos producidos por el Estado para hacer legible la sociedad, y deberían ser estudiados como lo han sido los censos o los mapas: no como objetos neutrales, sino como mecanismos atravesados por relaciones de poder, mecanismos que constituyen y son parte de culturas y sociedades y que producen textos de los que emana autoridad. Son parte del archivo estatal y, por lo tanto, sus materiales son producto de un proceso cuidadoso de selección y organización: no

${ }^{2}$ Es importante distinguir entre estas comisiones y estos comités formados ad hoc, y los que funcionan de manera permanente en el periodo poscolonial. Ejemplos de estos últimos son la Comisión Planificadora o el Comité sobre Industria.

${ }^{3}$ El comité fue formado en 1959 y produjo su reporte en 1962.

${ }^{4}$ Algunos ejemplos que dan idea de la amplitud de su uso son: la Real Comisión para Palestina (1936), para estudiar los episodios de violencia y ver si en el Mandato se estaban cumpliendo las obligaciones contraídas hacia árabes y judíos; la Real Comisión de Investigación sobre Tráfico de Drogas en Australia (1981-1983) y la Comisión para el Esclarecimiento Histórico (1994) en Guatemala para indagar en torno a violencia $\mathrm{y}$ violaciones a derechos humanos. 
todo es material de archivo, como nos recuerda Gyanendra Pandey (2012, p. 37), sólo lo que es considerado útil, racional, relevante.

Por otro lado, planteo contribuir a la creciente literatura que ha examinado los primeros años después de la independencia de países como India. Los autores de esos trabajos se han abocado a examinar procesos de descolonización, como Dipesh Chakrabarty (2011), quien estudió la Conferencia de Bandung y resaltó las convergencias y las diferencias entre los líderes de los países asistentes, así como los debates entre ellos sobre la relación con la modernización y las exmetrópolis. Sunil S. Amrith (2006) se ha interesado, entre otros temas, en las políticas de salud y nutrición, vinculando lo nacional y lo internacional. Así, realizó un estudio sobre descolonización y salud que abarca las décadas de 1930 a 1960, cuando el Imperio británico estaba en crisis. En su recuento, sobresalen médicos que impulsaban una medicina social, fundaciones (por ejemplo, la Fundación Rockefeller) y nacientes organizaciones internacionales. Asimismo, trazó un cuadro muy completo de la nutrición en la primera mitad del siglo xx y reflexionó sobre la política de salud pública en el periodo independiente (2007, 2008). Este último texto es particularmente interesante, pues explora las varias corrientes que convergen en la concepción de la salud y la forma en que han dado resultados muy poco satisfactorios.

Asimismo, varios autores han reflexionado sobre las rupturas y las continuidades entre los periodos colonial y poscolonial para ofrecer visiones más complejas, empezando por el propio Chakrabarty (2011). Por ejemplo, en su libro dedicado a India independiente, Bipan Chandra, Mridula Mukherjee y Aditya Mukherjee (2002) establecen que el gobierno independiente conservó ciertas estructuras y decisiones del periodo colonial no por una cuestión de simple continuidad, sino porque eran resultado de las críticas al gobierno colonial, de la lucha de los varios movimientos nacionalistas. Un caso es el legado constitucional del periodo colonial (Chandra et al., 2002, p. 33). Tay- 
lor Sherman (2013) afirma que muchos autores descuidaron el análisis de los primeros años de vida independiente y prefirieron empezar con los planes quinquenales, en especial con el segundo. Ella analizó la política alimentaria y educativa de esos primeros años y señala que muchas decisiones importantes fueron tomadas en ese momento (Sherman, 2013, 2018). Sostiene, asimismo, que si bien la modernización era una aspiración importante, también lo era distinguirse del gobierno colonial, aunque esto no siempre fuera fácil (2013, p. 572). Macmillen y Brimnes (2010) estudiaron la forma en que la modernización, presente en varios proyectos e instituciones, fue entendida durante los primeros años de India independiente. Para ello analizaron la resistencia a las campañas de vacunación masiva y encontraron que, notablemente, el discurso empleado no tenía elementos antioccidentales o anticientíficos, sino que mostraba un rechazo hacia lo que se percibía como modernización de "segunda clase": en vez de impulsar un cambio radical y mejorar las condiciones de vida de la población, se introdujo la vacuna, es decir, un mero recurso tecnológico.

En diálogo con estos trabajos, este texto propone una mirada crítica, desde la historia social, al Comité Mudaliar y a la política de salud de los primeros años de independencia. Comienzo analizando las características de las comisiones y los comités en India colonial y poscolonial, y luego señalo algunos procesos históricos relevantes del contexto de formación, para posteriormente analizar la composición del Comité Mudaliar y su reporte. Para ello, utilizo los reportes de varios comités de salud, con énfasis en el último (Bhore) de la época colonial y el primero (Mudaliar) de la época poscolonial. Asimismo, echo mano de literatura y documentos clave para los primeros años de India independiente, principalmente la Constitución $\mathrm{y}$ los planes quinquenales. 


\section{Comisiones y comités}

Aunque he usado los términos "comisiones" y "comités" como si fueran equivalentes, en realidad tenían diferencias. Su origen se remonta a Gran Bretaña, donde las comisiones eran nombradas por la Corona, pero eran vistas como independientes. Además, celebraban audiencias públicas generalmente. En cambio, los comités podían llegar a su fin por órdenes de autoridades superiores y eran usados cuando los temas tenían que ver con presupuesto, porque no celebraban audiencias públicas y así evitaban situaciones incómodas (Gosnell, 1934, pp. 85-86).

Estos mecanismos fueron creados por el Estado inglés para recabar información de diversos tipos y forman parte de su proceso de conformación y expansión, como lo estudiaron Phillip Corrigan y Derek Sayer (1985, p. 70). Tanto comisiones como comités poseían "términos de referencia" que incluían sus objetivos y sus alcances precisos (Ashforth, 1990, p. 9; Gosnell, 1934, pp. 88-90).

En cuanto a sus integrantes, podían ser expertos en el tema a tratar, pero también podían ser representantes de sectores involucrados (aunque actuando de forma imparcial) y funcionarios. Los dirigían generalmente altos funcionarios nombrados por la Corona (Ashforth, 1990, p. 5).

En India colonial, comisiones y comités compartieron estas características y fueron establecidos para tomar decisiones y evaluar las actividades gubernamentales, especialmente en tiempos de crisis (Hansen, 2000, p. 39). Al revisar el periodo colonial, la lista es larga y su ámbito de incidencia, enorme. Así encontramos la Comisión de la Lepra en 1890, la Comisión Estatutaria (Comisión Simon) en 1927, la Real Comisión del Trabajo en 1929 y la Comisión Fronteriza en 1947.

En India independiente, la presencia de comisiones y comités ad hoc ha sido constante, lo cual nos obliga a pensar en los lazos entre el periodo colonial y el poscolonial. Es interesante que se haya mantenido un mecanismo que durante la Colo- 
nia fue criticado muchas veces por su falta de resultados. Sin embargo, hay que considerar que tanto las comisiones como los comités se volvieron espacios para la participación y el debate, así como blancos de la crítica y figuras de cohesión de la opinión pública.

En 1952, se publicó el Act on Inquiry Commissions, que delineaba las características básicas de las comisiones y los comités, varias de las cuales provenían de la época colonial, mientras que otras eran nuevas. ${ }^{5} \mathrm{El}$ documento establece que el gobierno central (Parlamento) o los gobiernos estatales (legislaturas estatales) pueden nombrar una comisión de investigación para recabar la información necesaria con el fin de definir la política en cualquier tema. Estos mecanismos tienen cierto número de integrantes, entre los que se incluye la persona que los encabeza, y pueden tener asesores. Ellos elaboran un reporte que puede o no ser publicado e impactar la política gubernamental, incluso si ésa fue la motivación original. Asimismo, la comisión decide si lleva a cabo audiencias públicas o privadas, pero el gobierno central puede pedirle que sean privadas.

En nuestro caso, el Comité Mudaliar se integró siguiendo el patrón arriba descrito. Estuvo compuesto por expertos independientes del Estado, algo que le daría legitimidad y perspectiva crítica. Sin embargo, la capacidad de juicio de éste o cualquier otro comité está enmarcada por los términos de referencia, no importa si hablamos del periodo colonial o del poscolonial (Ashforth, 1990, p. 9; Gosnell, 1934, pp. 88-90). La incapacidad para ir más allá de estos términos provoca insatisfacción en torno a comisiones y comités hasta el día de hoy: existe la sensación de que son mecanismos inútiles que absorben tiempo y recursos, sin rendir los frutos esperados (dependiendo del tema investigado). De manera que estudiar una comisión o un comité implica dar cuenta de las respuestas de la sociedad, pero sin perder de vista el proceso de generación de conocimiento por parte del Estado.

\footnotetext{
${ }^{5} \mathrm{El}$ acta ha sido enmendada varias veces desde su aprobación.
} 
Las comisiones y los comités involucran algunas de las siguientes tareas: audiencias públicas con partes interesadas, recorridos de los integrantes, aplicación de cuestionarios, petición de información al gobierno, convocatoria para el envío de memorandos con información o planteamientos sobre el tema en cuestión, reuniones entre integrantes y un largo proceso de redacción del reporte, que es publicado posteriormente. ${ }^{6}$ Es de esperar que, después de tan prolongado procedimiento, la falta de resultados profundice la insatisfacción pública. Si pensamos en la larga lista de comités de salud del periodo independiente y los muchos problemas que hay hasta la fecha, compartiremos ese sentimiento de insatisfacción. ${ }^{7}$

\section{Comienzos}

Chandra, Mukherjee y Mukherjee (2002) describieron los “años iniciales" como un periodo marcado por múltiples desafíos: la compleja y complicada incorporación de principados a India (o a Pakistán), las dificultades planteadas por los refugiados, la violencia comunalista y la relación con Pakistán, además de las tareas cotidianas de administrar un país: "la puesta en marcha de un sistema administrativo, amenazado con la des-

${ }^{6}$ Hay comisiones y comités cuyos temas atacan directamente la legitimidad del Estado, por lo que sus reportes no circulan entre el público, sino parcialmente, o se conocen a través de noticias periodísticas. Dos ejemplos son la Comisión Nanavati-Mehta y la Comisión Banerjee, que investigaron la violencia ejercida sobre los musulmanes en Godhra, Gujarat, en 2002 (Martínez, 2019, pp. 121-122).

7 Si tomamos algunos indicadores relacionados con la salud de la población india, se tendrá una idea más clara de los problemas que la aquejan. De acuerdo con la oms, India tiene una expectativa de vida de 67/70 años (2016), una probabilidad de mortalidad de 37 por cada 1000 en niños menores de cinco años (2018) y una probabilidad de mortalidad de 214/138 por cada 1000 personas de 15 a 60 años (2016). Su gasto en salud es de 4.7\% del PIB (2014) (World Health Organization [wHO], 2020a). Para efectos de comparación, México tiene una expectativa de vida de 74/79 años (2016), una probabilidad de mortalidad de 13 por cada 1000 en niños menores de cinco años (2018) y una probabilidad de mortalidad de $164 / 89$ por cada 1000 personas de 15 a 60 años (2016). Finalmente, su gasto en salud es de 6.3\% del PIB (WHO, 2020b). 
composición debido a la Partición y a la división ilógica del ejército y de la alta burocracia, en la práctica, de acuerdo con la comunidad religiosa" (p. 69).

La idea de que el Estado debía desempeñar un papel importante en el desarrollo económico de India se remonta a finales del siglo XIX, y fue retomada en la Resolución de Karachi del Partido del Congreso (1931) y puesta en práctica en 1938, cuando estableció un Comité de Planificación Nacional, pero sus trabajos fueron interrumpidos primero cuando, en 1940, Nehru fue enviado a prisión por el gobierno colonial, y, posteriormente, por la expansión japonesa durante la Segunda Guerra Mundial. Finalmente, tras la independencia, se estableció la Comisión Planificadora en marzo de 1950 (Chandra et al., 2002, pp. 341-344).

Como se mencionó, varios autores han escrito sobre la compleja relación entre lo colonial y lo poscolonial y la importancia de reflexionar acerca de la relación entre ambos periodos (Chandra et al., 2002; Sherman, 2013). Cuando se estableció el mencionado Comité de Planificación Nacional de 1938, se integró un subcomité encargado de salud que trabajó sólo durante un tiempo (Sokhey, 1948).

En 1943, el gobierno británico integró un Comité de Salud y Desarrollo (Health Survey and Development Committee), mejor conocido como el Comité Bhore, que publicó su reporte en 1946. El objetivo era hacer una evaluación general y plantear recomendaciones, pues el Gobierno de India necesitaba estar preparado para tomar decisiones informadas tan pronto como la guerra terminara, para así organizar las labores de reconstrucción (Bhore, 1946, I, pp. 1-2). Es interesante que cuando el subcomité Sokhey reanudó sus labores en 1946, reconoció la relevancia del reporte del Comité Bhore para armar una política nacional coordinada que delineara la organización y la administración necesarias (Sokhey, 1948, pp. 143-144).

Una vez que se obtuvo la independencia en 1947, las recomendaciones del Reporte Bhore se convirtieron en la guía para el nuevo gobierno. Esta decisión no es sorprendente, pues como 
el propio subcomité Sokhey había señalado, los integrantes del Comité Bhore eran expertos que habían hecho un trabajo minucioso: todos (excepto Josep Bhore) eran médicos, investigadores o funcionarios en áreas relacionadas; sin olvidar que habían contado con la asesoría de expertos de Gran Bretaña, Estados Unidos, Australia y la Unión Soviética (Bhore, 1946, I, pp. ii, 5; Sokhey, 1948, pp. 143-144). Para Borkar, quien escribió sobre los logros en salud del gobierno independiente, no había duda de por qué el Reporte Bhore era su guía: sus términos de referencia habían sido vastos y sus recomendaciones eran de tal trascendencia que, quizá, no habrían sido puestas en marcha por el gobierno británico de haber continuado en el poder (Borkar, 1957, p. 6). Esta última y aguda observación de Borkar apunta a la falta de capacidad del gobierno colonial para hacerse cargo del bienestar de la población india, una de las piedras angulares para justificar su presencia.

El Reporte Bhore se publicó en tres volúmenes. El primer volumen describía el estado de la salud pública y su organización. El segundo contenía las recomendaciones del comité. El tercero incluía gran cantidad de datos: desde el número de camas de hospital por habitante hasta las cifras de mortalidad y morbilidad.

El primer volumen presentaba al lector una imagen desalentadora de la salud y de los problemas que pronto enfrentaría el gobierno independiente. Tres indicadores en particular (tasa de mortalidad, tasa de mortalidad infantil y esperanza de vida) mostraban que India británica tenía un rezago importante no sólo respecto a la metrópoli (lo que era esperable), sino también respecto a los asentamientos británicos, con excepción de los Asentamientos del Estrecho y de Egipto, que también tenían cifras negativas. El cuadro 1 permite establecer una comparación entre varios países e India, y comprender la gravedad de la situación. 
CuAdro 1. Estado de la salud en India británica

\begin{tabular}{|c|c|c|c|c|}
\hline \multirow[b]{2}{*}{ País } & \multirow{2}{*}{$\begin{array}{c}\text { Tasa } \\
\text { de } \\
\text { mortalidad } \\
\text { (1937) }\end{array}$} & \multirow{2}{*}{$\begin{array}{c}\text { Tasa } \\
\text { de } \\
\text { mortalidad } \\
\text { infantil } \\
\text { (1937) }\end{array}$} & \multicolumn{2}{|c|}{$\begin{array}{c}\text { Esperanza } \\
\text { de vida }\end{array}$} \\
\hline & & & Hombres & Mujeres \\
\hline Canadá & 10.2 & 76 & 59.32 & 61 (1929-31) \\
\hline Inglaterra y Gales & 12.4 & 58 & 58.74 & $62.88(1930-32)$ \\
\hline $\begin{array}{l}\text { Federación } \\
\text { de Estados Malayos }\end{array}$ & 19.9 & 147 & & \\
\hline India británica & 22.4 & 162 & 26.91 & $26.56(1921-30)$ \\
\hline $\begin{array}{r}\text { Asentamientos } \\
\text { del Estrecho }\end{array}$ & 22.5 & 156 & & \\
\hline Egipto & 27.2 & 165 & & \\
\hline
\end{tabular}

Fuente: elaboración propia con base en "Table 1: A comparison of Indian mortality rates and expectation of life with those of certain other countries", en Bhore, 1946, I, pp. 7-8.

El estudio realizado por el Comité Bhore mostró otras áreas preocupantes, todas relacionadas con problemas profundos. Las condiciones difíciles en las cuales vivían las mujeres en edad reproductiva redundaban en una elevada mortalidad materna. La presencia y las consecuencias de enfermedades como malaria, cólera y viruela se reflejaban en muertes anuales. Pero también había muertes atribuidas a factores poco precisos, como "fiebres" y "otras causas" (Bhore, 1946, I, p. 10).

El reporte apuntaba a varios factores detrás de estos problemas. La pobre nutrición y las deficiencias en salud ambiental, así como una falta de educación en higiene, hacían que las enfermedades afectaran a la población. El número limitado de dispensarios, hospitales y personal capacitado para la prevención y la cura de pacientes era una constante a lo largo y ancho de India británica (Bhore, 1946, I, pp. 11-20). 
Parecía imposible echar a andar las medidas necesarias para remontar esta situación. Por ejemplo, si vemos las cifras relacionadas con el personal médico, la información indica que había un médico por cada 6300 personas, mientras que en el Reino Unido había uno por cada 1000. De acuerdo con el Comité Bhore, si se planteaba tener una proporción de al menos un médico por cada 2000 personas para 1971, se requerían 185000 médicos. Aun así, la proporción sería menor que la encontrada en Reino Unido, pero, afirmaba el reporte, debido a la magnitud del problema en India, significaría un logro importante y un esfuerzo sin precedentes (Bhore, 1946, I, p. 13).

Como se ve, el gobierno independiente enfrentaba una situación compleja. Por un lado, una parte de las recomendaciones del segundo volumen fue adoptada por el Primer Plan Quinquenal (1951-1956) y se tradujo en la creación de un sistema de salud que aspiraba a cubrir desde cuidado básico hasta atención especializada. A la red del periodo colonial de dispensarios, hospitales, institutos de investigación y educación médica, entre otros, se unieron nuevas instituciones, como el All-India Institute of Medical Science, que fue fundado en 1956, para fortalecer el área de enseñanza de la medicina a nivel tanto de licenciatura como de posgrado.

En términos de organización, se entendía que las principales responsabilidades de cuidado de la salud estaban en manos de los gobiernos estatales, mientras que la enseñanza y la investigación eran responsabilidad del gobierno central. Esto significaba que había un Consejo Central de Salud, dirigido por el Ministerio de Salud Central, al frente del cual estaba Rajkumari Amrit Kaur, y que incluía a los ministros de Salud de cada estado (Planning Commission, 1951, Ch. 32: Health). Sin embargo, hacía falta mucho más que sólo coordinar políticas.

Por otro lado, los problemas urgentes obligaron al gobierno a dedicar tiempo y recursos a las personas afectadas por la Partición: campamentos para desplazados, atención médica y 
operaciones de rescate. Amrith (2006, p. 79) señaló que, si el Reporte Bhore había expuesto las dificultades en términos de infraestructura colonial, la Partición las había confirmado en la práctica.

Incluso si el periodo que va de 1951 a 1964 mostró algunos logros ${ }^{8}$ la conformación del Estado hizo que la salud pasara a segundo plano, con lo que se apartó del tono de urgencia y centralidad que se encontraba en el Reporte Bhore. ${ }^{9}$ Tareas como elaborar la Constitución o integrar la Comisión Nacional Planificadora absorbieron la mayor parte del tiempo y de los recursos: esto se reflejó en que la salud fuera considerada una tarea de los gobiernos estatales. Así, el plan original del Comité Bhore, que contemplaba dos series de recomendaciones, una a corto plazo (5 a 10 años) y otra a largo plazo, dio paso a arreglos que eran, sobre todo, de corto plazo (Bhore, 1946, II, p. 4).

Lo más importante era que las líneas iniciales del capítulo de salud del Primer Plan redujeron los problemas que enfrentaba India a una cuestión de pérdida económica. Incluso si la salud se definía no sólo como la ausencia de enfermedad, sino más en términos de calidad de vida y bienestar general, era vista como un recurso: el más importante, pero finalmente un recurso para el desarrollo económico (Planning Commission, 1951, Ch. 32: Health). El Reporte Bhore había contribuido enormemente al concebir la salud como una responsabilidad del Estado y no de voluntarios u organizaciones caritativas, pero había dado énfasis a la idea de la salud como un recurso económico, incluso si parte de sus integrantes la concebían como un derecho (Amrith, 2006, pp. 62-63; Murthy, Sarin y Jain, 2013, pp. 75-76).

${ }^{8}$ Un par de ejemplos ilustran bien esto: se reconstituyó el Indian Council of Medical Research (el nombre anterior era Indian Research Fund Association), se amplió su área de impacto y se lanzó el Programa Nacional para el Control de la Malaria (Borkar, 1957, pp. 75, 86).

${ }_{9}$ Amrith (2006, p. 81) ve en esta decisión de poner la salud en manos de gobiernos estatales una tendencia a conservar algunos elementos del gobierno colonial, entre ellos, la estructura fragmentada. 
El Segundo Plan (1956-1961) fue más crítico y directo. Simplemente estableció que aspiraba a expandir y mejorar los servicios de salud. En parte, esto se debía a que algunos de los problemas más urgentes planteados por la Independencia y la Partición ya estaban resueltos o habían adquirido una naturaleza permanente que requería una perspectiva distinta. Y, en parte, también, porque no se había progresado mucho desde el Primer Plan.

Así, por ejemplo, en 1951 había 8600 hospitales con 113000 camas, y hacia el final del Primer Plan había 10000 hospitales con 125000 camas (Planning Commission, 1956, Ch. 25: Health).

Aunque había habido cierta mejoría, se estaba muy lejos de la meta de una cama de hospital por cada 1000 personas: la población en 1951 era de 361 millones, que habría requerido aproximadamente 361000 camas de hospital. Se observaba una situación similar en relación con el personal médico: el número de doctores, enfermeros, visitadores médicos y otros más estaba por debajo de lo esperado.

En este contexto, el gobierno indio decidió nombrar un comité que haría una evaluación de la salud de la población durante los primeros años de vida independiente, así como recomendaciones.

\section{Evaluación de los comienzos}

Chakrabarty (2011) escribió que los estados en proceso de descolonización desarrollaron un discurso marcado por dos estilos, el pedagógico y el dialógico (pp. 209-210). El estilo pedagógico aspiraba a modernizar el país, a emparejarse con Occidente. Los líderes independientes necesitaban enseñar a sus compatriotas cómo ser ciudadanos y a comportarse de acuerdo con ello. Así, era importante instruirlos sobre cuándo y cómo entonar el himno nacional (pp. 209-211), pero también 
explicarles los esfuerzos del gobierno para mejorar la salud de la población. En 1957, dos años antes del nombramiento del Comité Mudaliar, el gobierno pidió a G. Borkar que elaborara un reporte del estado de la salud, sólo que en esa ocasión el libro estaba pensado para un público más amplio, puesto que, de acuerdo con el propio Jawaharlal Nehru, había sido "escrito en un estilo popular" (Borkar, 1957, p. v).

El libro está dividido en cinco partes y empieza estableciendo una diferencia central respecto a la administración colonial: mientras que ésta se afanaba por mantener la ley y el orden y defender el imperio, el Estado independiente tenía como tarea primordial hacer a su población feliz y saludable (Borkar, 1957, pp. xxii, xxv).

Tras describir las condiciones terribles en que se encontraba India, las restantes cuatro partes describen, como si se tratara de una guerra, las tareas por realizar. Palabras como "bases y armamento", "campañas y operaciones", "líneas enemigas" y "resistencia" en títulos y subtítulos transmiten la sensación de que había una unidad de propósito y determinación. Estamos ante un texto que claramente estaba pensando para educar: describía los logros de los primeros años, las tareas desarrolladas por los expertos y la ruta a seguir.

Es preciso ver al Comité Mudaliar en este contexto, marcado por la convicción y una actitud crítica. El Ministerio de Salud lo nombró en 1959 y estableció que sus términos de referencia incluían hacer un estudio sobre las condiciones de salud desde la publicación del Reporte Bhore (1946), evaluar los planes quinquenales primero y segundo y hacer recomendaciones (Mudaliar, 1962, p. 7).

Que fuera un comité el mecanismo elegido por el gobierno indio y no una comisión habla de las tensiones en torno al tratamiento del tema. Se esperaba que un comité recopilara información a través de diversos canales y produjera recomendaciones, pero no tenía la misma visibilidad que una comisión, la cual poseía las características de una corte civil (Government of India, 1952). 
A pesar de ello, dos elementos destacan en este comité. Uno es la relevancia de sus objetivos: llevar a cabo un estudio minucioso desde 1946. El otro fue su autonomía. A diferencia de los reportes producidos por comités posteriores, el Reporte Mudaliar afirmó su independencia enérgicamente. En las primeras páginas, Mudaliar agradeció al gobierno indio el ofrecimiento de apoyo, pero afirmó que no comprometería su independencia (Mudaliar, 1962, p. 10). El reporte revisó de forma crítica los dos primeros planes quinquenales, especialmente el presupuesto asignado a salud, y los contrastó con el documento que contenía la guía, el Reporte Bhore.

El Comité Mudaliar tenía 16 integrantes, que en su mayoría estaban relacionados, de una forma u otra, con el campo de la salud. Como se mencionó, A. Lakshmanaswami Mudaliar, quien encabezó el comité, era médico, con experiencia en instituciones educativas en medicina, había participado en el Comité Bhore y presidía el Comité Ejecutivo de la OMs. En cuanto al resto de los integrantes, la mayoría se había formado en medicina, la cual combinaban con la investigación o con la administración en alguna institución de salud.

Ashforth $(1990$, p. 5) propone varias razones para entender por qué una comisión o comité es nombrado, y una de ellas es la idea de reunir a un grupo de expertos que aspiran a trascender lo político. La noción anterior se confirma al revisar la lista de integrantes del Comité Mudaliar (Mudaliar, 1962, pp. 7-9). Así, por ejemplo, tenemos a V. Srinivasan, director general de Servicios de Salud, Ministerio de Salud, Nueva Delhi; a K. N. Rao, director de Servicios Médicos, Hyderabad; al doctor C. O. Karunakaran, expresidente de la Asociación Médica India, Trivandrum, y a la doctora Hilda M. Lazarus, profesora de obstetricia y ginecología, K. G. Medical College, Visakhapatnam.

El tipo de membresía nos habla de la confianza depositada en los expertos. Timothy Mitchell (2002, p. 15) escribió sobre la participación que los higienistas públicos, los administradores, los cartógrafos y los ingenieros tuvieron en el proceso 
de modernización en Egipto, y planteó una pregunta crucial: ¿cómo es que se convirtieron en los portavoces de la modernidad, del desarrollo, de la racionalidad? En el caso de India, los médicos y los administradores en puestos de salud pública habían pasado los últimos años practicando la medicina, haciendo investigación, dirigiendo institutos y poniendo mucho de esto en papel: reportes anuales, proyectos de desarrollo, artículos en revistas, ponencias en congresos o publicaciones para el público en general. Entre todos estos escritos, es posible encontrar estudios que hacían más entendibles los problemas de salud enfrentados por India: eso es lo que se esperaba de la alta especialización y de la razón, nos recuerda Mitchell (pp. 34, 37).

Además de ello, es importante añadir el papel de los expertos en la consolidación de profesiones e instituciones. Veamos el ejemplo de Hilda M. Lazarus. Desde el siglo XIX se registró una demanda de atención y educación médica para las mujeres indias, que se consolidó hacia las décadas de 1920 y 1930 (Burton, 1996; Forbes, 1994 y 2004, pp. 161-166). Entre las mujeres indias que estudiaron medicina, la doctora Lazarus fue parte tanto del Comité Bhore como del Mudaliar. Lazarus estudió en el Medical College, en Madrás, y posteriormente fue nombrada asistente en ginecología y obstetricia en el Lady Hardinge Medical College Hospital, en Delhi. Geraldine Forbes (2004, pp. 163-164) nos recuerda que Lazarus fue la primera mujer india en ser parte del Women's Medical Service, en el cual estuvo durante 30 años, y que dirigió instituciones, además de formar a muchos profesionales en el cuidado de la salud.

El Reporte Mudaliar reconocía la enormidad de los desafíos en salud y los cambios efectuados por la Independencia. En primer lugar, aunque el Comité Bhore había producido un texto notable, no contenía información para toda India. Cuando se había realizado el estudio, entre 1943 y 1946, se había hecho en India británica, los principados no habían sido incluidos. A partir de 1947 habían ocurrido cambios en el territorio que 
habían tenido impacto en la información necesaria para los planes quinquenales (Mudaliar, 1962, pp. 29-30).

Otra diferencia es que Nehru había introducido la planificación. El gobierno indio emitió una Resolución, en 1950, que afirmaba la necesidad de planear cuidadosamente, de manera que el país se desarrollara. Parafraseando la Resolución, los planes formulados por la Comisión Planificadora se basarían en información precisa sobre los recursos disponibles, y, haciendo una abstracción de los problemas cotidianos, se enfocarían en generar crecimiento económico en la vida de los ciudadanos (Government of India, 1950).

El comité se organizó en seis subcomités que trabajarían en temas específicos: enseñanza e instituciones de investigación, atención médica en áreas rurales y urbanas, salud pública, planificación familiar y medicamentos. El comité principal se reuniría en ciertas ocasiones y los subcomités recorrerían el país para tener una mejor idea de los problemas. Además, el comité recopilaría información a través de cuestionarios, visitas a instituciones importantes, reuniones con representantes de organizaciones o con individuos y memorandos recibidos (Mudaliar, 1962, p. 11).

El reporte del Comité Mudaliar fue crítico de las restricciones presupuestales sobre salud y de la falta de coordinación entre los gobiernos central y estatales, pero no fue suficientemente lejos. Por ejemplo, no había necesidad, estableció el reporte, de hacer ningún cambio a alguna provisión de la Constitución ( $\mathrm{Mu}-$ daliar, 1962, p. 465). El resultado fue un reporte rico en detalles y recomendaciones, pero con incongruencias internas.

Veamos dos elementos centrales: presupuesto y organización administrativa. El reporte mostraba que el porcentaje dedicado a salud se había reducido: en el primer plan (1951-1956) había sido de 5.9\%, y en el segundo (1956-1961), de 5.5\%. Al consultar el tercer plan (1961-1966), el comité vio cómo el presupuesto se había reducido incluso más: a $4.25 \%$ del total, a pesar de la recomendación del Consejo Central de Salud de que debía darse 10\% (Mudaliar, 1962, pp. 30-33). 
En cuanto a la estructura administrativa, el reporte continúa: el artículo 263 de la Constitución había creado un Consejo Central de Salud, encabezado por el ministro de Salud de la Unión y con los ministros de Salud estatales como miembros. Sin embargo, el Consejo carecía de la capacidad para organizar las tareas nacionales de salud y de impacto sobre decisiones, pues funcionaba más como un foro que como una agencia para establecer la política nacional (Mudaliar, 1962, p. 464).

La larga lista de recomendaciones al final del reporte cubrió todos los temas posibles: desde los salarios de enfermeras y el número de camas en hospitales hasta la estructura administrativa requerida. La primera y más obvia era acerca del momento en que se elaboraban los planes quinquenales: Mudaliar y los otros miembros del comité señalaron que los planes eran elaborados una vez iniciado su periodo de cobertura, y esto tenía consecuencias negativas, pues no había tiempo suficiente para desarrollar el plan, los temas urgentes no eran tomados en cuenta, y cuando los planes nacionales eran finalmente puestos en marcha, los costos de los proyectos específicos se habían elevado. Sorprendentemente, ni siquiera los problemas urgentes y muy conocidos, como el drenaje o el abastecimiento de agua, recibían atención, hasta que el tema era incluido en el nuevo plan (Mudaliar, 1962, p. 478).

Tres recomendaciones importantes muestran las tensiones en el texto. La primera es que un elemento central era fortalecer en la Constitución la posición del Consejo de Salud, para que tuviera mayor impacto en términos tanto de asignación de fondos como de organización de actividades. Sin embargo, en el resumen de recomendaciones, el reporte sólo mencionaba la introducción de fondos de asistencia (grants-in-aid) del gobierno central en proyectos que tenían impacto nacional y la formación de un Sistema de Salud Nacional, entre otras cosas (Mudaliar, 1962, n.p.).

La segunda es que, a pesar de la relevancia de la salud, descrita como una promesa para los "ciudadanos" de la nueva nación 
(Mudaliar 1962, p. 477), el Reporte Mudaliar no la concibió como "derecho" y consideró que era inevitable pagar por la atención médica. Por ejemplo, recomendó que los hospitales cobraran a pacientes hospitalizados y ambulatorios (Mudaliar, 1962, pp. 78-79). ${ }^{10}$

La tercera es que el Reporte Mudaliar dejó de apoyar la idea de los centros de salud primarios, como se habían descrito en el Reporte Bhore, y recomendó que no se abrieran. La razón es que no estaban recibiendo suficientes fondos para equiparlos y contratar el número adecuado de personal. Pero, en vez de recomendar que el gobierno vigilara su buen funcionamiento, sugirió que no se abriera ninguno nuevo, a menos que tuviera las características delineadas en 1946. En el resumen de recomendaciones, el reporte afirmó que sería preferible abrir unidades móviles que brindaran atención médica al resto de la población rural (Mudaliar, 1962, pp. 3-4).

Al destacar estos elementos, traté de mostrar las tensiones dentro del comité, que se veía a sí mismo como independiente y crítico, pero que debió hacer algunos compromisos en vista de las dificultades financieras y administrativas.

\section{El paso de los años}

Esta forma de trabajar con base en comisiones o comités de investigación se ha vuelto una característica del Estado indio. Su capacidad para organizar la realidad y producir estudios que la condensen ha probado ser valiosa y se asemeja a otros mecanismos usados por el Estado para gobernar (Scott, 1998). Al mismo tiempo, los momentos en los que divide sus labores han terminado por ser muy conocidos y permiten que la población sepa qué esperar: hay una constitución del organismo, una

${ }^{10}$ Este razonamiento dejaba de lado el hecho de que una familia podría empobrecerse debido a los gastos por una enfermedad. Este elemento se encontró décadas después en otra evaluación gubernamental realizada por Chidambaram y Ramadoss (2005). 
búsqueda de información acompañada en ocasiones de audiencias, un reporte y toma de decisiones. La propuesta de Ashforth (1990) de identificar tres momentos en las labores de una comisión o un comité es útil y sugerente por desvelar atinadamente las relaciones de poder. En el primer momento, que denomina investigativo, las personas nombradas por el Estado interactúan con aquellos que representan intereses sociales específicos. Este procedimiento no sólo encarna en una forma de hacer preguntas de manera científica, rigurosa, sino que también constituye un "discurso social" (p. 7). El segundo momento, el persuasivo, está representado por el reporte: un texto con estrategias retóricas que es preciso analizar (p. 8). Los reportes no son simples resúmenes de una investigación: presentan la visión autorizada sobre un tema y el modo de lidiar con éste, como vimos con los reportes Bhore y Mudaliar. En el último momento, el del archivo, la comisión o el comité entra en diálogo con la historia. Los materiales pasan a ser parte del archivo: el conocimiento que han elaborado no es neutral, por supuesto, sino que está plenamente vinculado a las formaciones del poder estatal (p. 8).

En el ámbito de la salud, hasta este día, el gobierno indio ha nombrado varios comités y una comisión: Comité Chadha (1963), Comité Mukherjee (1965), Comité Mukherjee (1966), Comité Jungalwalla (1967), Comité Kartar Singh (1973), Comité Shrivastav (1975), Comité Bajaj (1986) y Comisión Nacional de Macroeconomía y Salud (2005). ${ }^{11}$ Sin embargo, ninguno ha sido tan ambicioso como el Comité Bhore o el Mudaliar, excepto quizá, la Comisión Nacional de Macroeconomía y Salud. ${ }^{12}$

${ }^{11}$ Los años indicados corresponden a la publicación de los reportes.

${ }^{12}$ La Comisión Nacional de Macroeconomía y Salud estuvo encabezada por dos personas (el ministro de Salud y Bienestar Familiar y el ministro de Finanzas) y recibió fondos y asesoría técnica de la oms (Chidambaram y Ramadoss, 2005, p. vii). El gobierno realizó otros estudios sobre el estado de salud de la población, pero ya no en forma de comisiones y comités ad hoc. La National Family Health Survey ha hecho varias rondas de estudios para proveer de información al Ministerio de Salud y Bienestar Familiar y ha recibido fondos de diversas agencias internacionales. La primera serie fue hecha en 1992 y 1993: los resultados, así como los de los siguientes estudios, están 
Los restantes han tenido términos de referencia muy limitados, como el análisis de programas para atención de enfermedades específicas, de proyectos o de estudios sobre planes de estudio para la enseñanza de la medicina.

Un rasgo más por destacar es la relevancia que han adquirido otras aproximaciones a la enfermedad y la salud: ayurveda, unani, siddha, naturopatía y homeopatía. ${ }^{13}$ De éstos, sólo los dos primeros "sistemas indígenas" (como fueron llamados en los textos estudiados) figuraron de forma marginal, y ni los vaid (sistema ayurveda) ni los hakim (sistema unani) formaron parte de los comités. ${ }^{14}$ Los reportes de Sokhey, Bhore y Mudaliar coincidían en que un sistema científico debía ser la base para la salud, y si bien preveían un periodo de transición en que los vaid y los hakim brindarían atención médica, para hacerlo posible debían recibir entrenamiento en medicina (Sokhey, 1948, pp. 22-23; Bhore, 1946, II, p. 461; Mudaliar, 1962, pp. 451-462). ${ }^{15}$ Sin embargo, con el tiempo, y una vez que la formación en medicina estuviera al alcance de todos, desaparecería la "confusión" entre los varios tipos de entrenamiento y de sistemas médicos (Sokhey, 1948, pp. 22-23).

El Comité Mudaliar agregó un elemento: India no podía quedarse atrasada respecto a otros países que habían hecho grandes progresos, además de que la ayuda recibida de organizaciones como la OMs apuntaba en ese sentido. ${ }^{16}$

\footnotetext{
disponibles para los investigadores en su página web (http://rchiips.org/nfhs/). La Misión Nacional para la Salud Rural se creó en 2005 para atender la falta de servicios en áreas rurales, y en 2013 se incorporó a la Misión de Salud Nacional. Por ejemplo, en 2011 la misión realizó un estudio en siete estados (Planning Commission, 2011).

${ }^{13}$ Agradezco a uno de los dictaminadores la sugerencia de hacer mención de este tema.

${ }^{14}$ El Comité Mudaliar incluyó a un médico, P. M. Mehta, interesado en los sistemas indígenas, particularmente en ayurveda. Para una introducción a estas aproximaciones a la salud, a su relación con el movimiento nacionalista, así como a la relevancia que han adquirido en la época independiente, véanse: Ernst, 2002; Ernst, 2007; Khan, 2005; Jaggi, 2000, y Mukharji, 2009.

${ }^{15}$ Sólo tres integrantes del Comité Bhore sugirieron que los vaid y los hakim podrían atender a la población.

${ }^{16}$ Estas posturas se han modificado paulatinamente. El Estado indio nombró en
} 


\section{Al final}

El propósito de este texto fue reflexionar sobre dos temas diferentes, aunque interrelacionados. Uno es la historia de las comisiones y los comités como mecanismo muy usado por el Estado indio. El otro son los proyectos estatales sobre salud en los primeros años del periodo independiente.

Las comisiones y los comités hablan de los objetivos, las contradicciones y los temores del Estado, así como de sus alcances: los memorandos enviados, las audiencias públicas, las tensiones entre sus integrantes y el seguimiento en la prensa apuntan a la presencia del Estado en la vida cotidiana.

Me enfoqué en el contexto, en las razones de Estado para llevar a cabo este tipo de estudios, en las tensiones en comisiones y comités y en la participación de los expertos. El análisis de los documentos elaborados por las comisiones y los comités señala las contradicciones inherentes en la política gubernamental vis à vis las decisiones prácticas tomadas. La compilación de datos a través de estudios cuidadosos y bien planeados fortalece la idea de que los procedimientos estatales son racionales y distinguen claramente los estados modernos de los premodernos (Scott, 1998, p. 51). Esta necesidad de diferenciarse fue muy evidente en los estados recién independizados.

Los reportes y otros documentos producidos por este régimen de expertos forman parte del archivo estatal de India, que nos habla de una historia incansable de esfuerzos por modernizar el país. Es importante pensarlos así: los incontables volúmenes producidos están pensados para hacer el mundo más simple y legible (aunque dejen de lado elementos relevantes), lo

\footnotetext{
paralelo comités de investigación para determinar cómo habría de hacerse la integración de sistemas indígenas con la medicina y destinó presupuesto para ellos en el primer y segundo planes. En 1995 se estableció un Departamento del Sistema Indio de Medicina y Homeopatía, cuyo nombre cambió en 2003 para finalmente adquirir la categoría de Ministerio en 2014 (Ministry of Ayush). La OMs (wHO, 2013) posee lineamientos y definiciones sobre las medicinas tradicionales y las medicinas complementarias, así como recomendaciones para su estudio y práctica.
} 
mismo que otras estrategias que el Estado usa para conocer a su población y su territorio. Uno de los mejores ejemplos, escribe Scott (1998, p. 47), son los mapas catastrales, que reflejan sobre todo el valor comercial de la tierra: "Cualquier otro valor que la tierra pudiera tener para propósitos de subsistencia o para la ecología local fue enmarcado como un valor estético, ritual o sentimental". Estudiar comisiones y comités, como cualquier otro tipo de material del archivo estatal, significa trabajar con el resultado de un proceso de selección y organización que ha dejado de lado cosas consideradas irrelevantes, innecesarias (Pandey, 2012).

Asimismo, deseo contribuir a una mejor comprensión de los primeros años del periodo poscolonial. Traté de mostrar que el paso de un periodo a otro no se reduce a rupturas absolutas ni a continuidades simples, sencillas. Que el reporte de un comité colonial, el Bhore, pudiera ser la base de la política en salud en el periodo independiente habla de los elementos relevantes que incluyó y de la crítica implícita al periodo colonial. A pesar de sus características progresistas, las dificultades de los primeros años y la reticencia a pensar en la salud como un derecho, no permitieron grandes avances. En cuanto al Comité Mudaliar, su desempeño buscó ser crítico y afirmó su independencia, pero sus recomendaciones se vieron limitadas por los múltiples problemas encontrados. Si bien es cierto que confirmó el papel del Estado como principal responsable de la salud de la población, no recomendó la modificación de la forma en que los gobiernos, central y estatales, se repartieron las tareas.

\section{Referencias}

Amrith, S. (2006). Decolonizing international bealth: India and Southeast Asia, 1930-65. Nueva York, NY: Palgrave Macmillan. Amrith, S. (2007). Political culture of health in India. A historical perspective. Economic and Political Weekly, 42(2), 114-121. 
Amrith, S. (2008). Food and welfare in India, c. 1900-1950. Comparative Studies in Society and History, 50(4), 1010-1035. https:// doi.org/10.1017/S001041750800042X

AsHFORTH, A. (1990). Reckoning schemes of legitimation: On commissions of inquiry as power/knowledge forms. Journal of Historical Sociology, 3(1), 1-22. https://doi.org/10.1111/j.1467-6443.1990. tb00143.x

Bajaj, J. S. (1986). Bajaj Committee Report. National Health Portal of India. Recuperado de https://www.nhp.gov.in/bajaj-committee-1986_pg

BHORE, J. (1946). Report of the Health Survey and Development Committee. Vol. I: Survey. Vol. II: Recommendations. Nueva Delhi: Manager of Publications. Recuperado de https://www.nhp.gov.in/ bhore-committee-1946_pg

Borkar, G. (1957). Health in independent India: A decade of progress. Nueva Delhi: Government of India, Ministry of Health.

Burton, A. (1996). Contesting the zenana. The mission to make lady doctors to India 1874-1885. Journal of British Studies, 35(3), 368-397.

Chadha, M. S. (1963). Chadha Committee Report. National Health Portal of India. Recuperado de https://www.nhp.gov.in/chadhacommittee-1963_pg

Chakrabarty, D. (2011). Los legados de Bandung: descolonización y política de la cultura. En S. Dube (Coord.), Encantamiento del desencantamiento: historias de la modernidad (pp. 199-223). México: El Colegio de México.

Chandra, B., Mukherjee, M. y Mukherjee, A. (2002). India after Independence: 1947-2000. Nueva Delhi: Penguin Books.

Chidambaram, P. y Ramadoss, A. (2005). Report of the National Commission on Macroeconomics and Health. Nueva Delhi: Government of India, Ministry of Health and Family Welfare. Recuperado de http://www.who.int/macrohealth/action/Report $\% 20$ of $\% 20$ the $\% 20$ National\%20Commission.pdf

CORRIGAN, P. y Sayer, D. (1985). The great arch: English state formation as cultural revolution. Oxford: Blackwell.

ERnst, W. (Ed.) (2002). Plural medicine: Tradition and modernity, 1800-2000. Londres: Routledge.

ERnst, W. (2007). Beyond East and West. From the history of colonial medicine to a social history of medicine(s) in South Asia. So- 
cial History of Medicine, 20(3), 505-524. https://doi.org/10.1093/ $\mathrm{shm} / \mathrm{hkm} 077$

Forbes, G. (1994). Medical careers and health care for Indian women: Patterns of control. Women's History Review, 3(4), 515-530.

Forbes, G. (2004). Women in modern India. Cambridge: Cambridge University Press.

Gosnell, H. F. (1934). British Royal Commissions of Inquiry. Political Science Quarterly, 49(1), 84-118. Recuperado de http:// www.psqonline.org/article.cfm?IDArticle $=4387$

Government of India (1950). Government of India's Resolution setting up the Planning Commission. Recuperado de https://niti. gov.in/planningcommission.gov.in/docs/aboutus/history/ PCresolution1950.pdf

Government of India (1952). The Commissions of Inquiry Act, 1952. Recuperado de http://legislative.gov.in/sites/default/files/ A1952-60.pdf

Hansen, T. B. (2000). Governance and myths of state in Mumbai. En J. C. Fuller y V. Bénéi (Eds.), The Everyday State and Society in Modern India (pp. 31-67). Nueva Delhi: Social Science Press.

JAGgi, O. P. (2000). Medicine in India: Modern period. Nueva York, NY: Oxford University Press.

Jungalwalla, N. (1967). Jungalwalla Report. National Health Portal of India. Recuperado de https://www.nhp.gov.in/jungalwallacommittee-1967_pg

KHAN, S. (2006). Systems of medicine and nationalist discourse in India: Towards "new horizons" in medical anthropology and history. Social Science E Medicine, 62(11), 2786-2797. https://doi. org/10.1016/j.socscimed.2005.10.039

Macmillen, Ch. W. y Brimnes, N. (2010). Medical modernization and medical nationalism: Resistance to mass tuberculosis vaccination in postcolonial India, 1948-1955. Comparative Studies in Society and History, 52(1), 180-209. https://doi.org/10.1017/ S0010417509990375

Martínez, B. (2019). Comisiones en pugna: hacia una explicación de la violencia de 2002 en India occidental. En W. Phillips, L. Carballido y Ó. Figueroa (Coords.), Inventar la India. Representaciones disciplinarias a partir de la historia, las humanidades y las 
artes (pp. 107-128). México: Universidad Nacional Autónoma de México.

Mitchell, T. (2002). Rule of experts. Egypt, techno-politics, modernity. Berkeley, CA: University of California Press.

Mudaliar, A. L. (1962). Report of the Health Survey and Planning Committee. National Health Portal of India. Recuperado de https://www.nhp.gov.in/mudaliar-committee-1962_pg

Mukharji, P. B. (2009). Pharmacology, 'indigenous knowledge', nationalism: A few words from the epitaph of subaltern science. En B. Pati y M. Harrison (Eds.), The social history of health and medicine in colonial India (pp. 195-212). Londres: Routledge.

Munherjee, B. (1965). Mukherjee Report. National Health Portal of India. Recuperado de https://www.nhp.gov.in/mukherjeecommittee-1965_pg

Mukherjee, B. (1966). Mukherjee Report. National Health Portal of India. Recuperado de https://www.nhp.gov.in/mukherjeecommittee-1966_pg

Murthy, P., Sarin, A. y Jain, S. (2013). International advisers to the Bhore Committee. Perceptions and visions for healthcare. Economic and Political Weekly, 48(10), 71-77.

Pandey, G. (2012). Un-archived histories: The "mad" and the "trifling”. Economic and Political Weekly, 47(1), 37-41.

Planning Commission (1951). First Five Year Plan (1951-1956). Nueva Delhi: Government of India. Recuperado de https://niti.gov.in/ planningcommission.gov.in/docs/plans/planrel/fiveyr/index1. html

Planning Commission (1956). Second Five Year Plan (1956-1961). Nueva Delhi: Government of India. https://niti.gov.in/ planningcommission.gov.in/docs/plans/planrel/fiveyr/index2. html

Planning Commission (2011). Evaluation Study of National Rural Health Mission (NRHM) in 7 States. Nueva Delhi: Government of India. Recuperado de https://nhm.gov.in/images/pdf/publication/Evaluation_study_of_NHM_in_7_States.pdf

ScotT, J. C. (1998). Seeing like a state: How certain schemes to improve the human condition have failed. New Haven, CT: Yale University Press.

Sherman, T. C. (2013). From 'grow more food' to 'miss a meal': Hunger, development and the limits of post-colonial nationalism 
in India, 1947-1957. South Asia: Journal of South Asian Studies, 36(4), 571-588. https://doi.org/10.1080/00856401.2013.833071 SHERMAN, T. C. (2018). Education in early postcolonial India: Expansion, experimentation and planned self-help. History of Education, 47(4), 504-520. https://doi.org/10.1080/0046760X.2017.1413214 SingH, K. (1973). Kartar Singh Committee Report. National Health Portal of India. Recuperado de https://www.nhp.gov.in/kartarsingh-committee-1973_pg

SoKHey, S. S. (1948). National Health. Bombay: Vora. Recuperado de http://www.communityhealth.in/ commun26/wiki/index. php?title=File:Sokhey_Committee_report_1948.PDF.pdf Srivastav, J. B. (1975). Srivastav Committee Report. Nueva Delhi: Government of India, National Health Portal. Recuperado de https://www.nhp.gov.in/shrivastav-committee-1975_pg wHO (Word Health Organization) (2013). WHO Traditional Medicine Strategy 2014-2023. Ginebra: World Health Organization.

wHO (Word Health Organization) (2020a). India [en línea]. Recuperado de https://www.who.int/countries/ind/en/

wHo (Word Health Organization) (2020b). Mexico [en línea]. Recuperado de https://www.who.int/countries/mex/en/

Laura Carballido Coria es profesora-investigadora en el Departamento de Ciencias Sociales de la Universidad Autónoma Metropolitana, Unidad Cuajimalpa. Es licenciada en historia por la Universidad Nacional Autónoma de México y maestra y doctora en estudios de Asia y África, área India, por El Colegio de México. Sus líneas de investigación, desde la perspectiva de la historia social y urbana, incluyen los procesos históricos de la Partición de India (1947), el comunalismo, los proyectos de salud pública en Delhi colonial y las comisiones de investigación (inquiry commissions). Ha realizado estancias de investigación en la Universidad Jawaharlal Nehru y en la Universidad de Oxford. 